\title{
Haemorrhagic breast infarction complicating anticoagulant therapy
}

\author{
S. BANIK* \\ M.B. B.S., D.Path., M.R.C.Path. \\ C. BRUN† \\ M.S., F.R.C.S. \\ Departments of *Pathology and $\uparrow$ Surgery, Royal Infirmary, Blackburn
}

\begin{abstract}
Summary
Haemorrhagic infarction of the breast induced by anticoagulant therapy is rare and has been infrequently reported in the literature. The following report illustrates such a case in which there was development of massive haemorrhagic infarction of the breast during treatment with nicoumalone for deep venous thrombosis after an operation for carcinoma of the colon.
\end{abstract}

\section{Introduction}

Oral anticoagulants are extensively used in the management of thrombo-embolic diseases and after cardiac surgery. Bleeding from the gum, skin bruises and haematuria are common side effects of anticoagulant drugs although cases of coumarininduced cutaneous necrosis are encountered rarely. Similarly, haemorrhagic infarction or necrosis of the breast complicating oral anticoagulant therapy is rare. Its pathogenesis is ill-understood and the clinical presentation can closely simulate that of a carcinoma of the breast.

\section{Case report}

A 65-year-old woman presented with pain in the left iliac fossa and alteration of bowel habit. Abdominal examination showed a mass in the left iliac fossa. A barium enema study confirmed the clinical diagnosis of carcinoma of the descending colon. A hemi-colectomy was performed, histological examination of which showed an adenocarcinoma with regional lymph node involvement.

On the 12th postoperative day she developed oedema of her right leg. Deep venous thrombosis was diagnosed and anticoagulant therapy was started with 3 doses of heparin, 12500 units given i.m. at 6-hourly intervals. At the same time, oral nicoumalone was administered, the dosage being $12 \mathrm{mg}, 8 \mathrm{mg}$ and $4 \mathrm{mg}$ on successive days. On the 4th day of anticoagulant therapy the patient suddenly developed a large haematoma in the right breast. The thrombotest was less than $5 \%$ and nicoumalone was stopped. The breast was extremely tender and oedematous with discoloration of the entire skin. This progressed to the develop- ment of a large, solid and non-fluctuant mass which occupied almost the entire breast. With the return of thrombotest within normal range, nicoumalone was re-administered in dosage varying from $2 \mathrm{mg}$ and $3 \mathrm{mg}$ daily. On account of persistent pain, tenderness and failure of the breast mass to resolve, a simple mastectomy was carried out 3 weeks later.

The resected specimen of the breast was very firm in consistency with bluish discoloration of the overlying skin. The cut surfaces showed haemorrhagic necrosis of the breast tissue. Histological examination showed interstitial haemorrhages, necrosis of the breast parenchyma and lipogranulomatous reaction (Fig. 1). No vascular thrombosis or arteritic changes were seen. There was no neoplasm in the breast.

\section{Comment}

The basic pathological change in the affected breast is a massive haemorrhagic infarction. Infarction of a fibro-adenoma of the breast has been well recognized as a clinicopathological entity (Haagensen, 1971). Pregnancy and lactation are the main predisposing factors, relative ischaemia being the basic denominator of these states. Spontaneous mammary infarction in the absence of a fibroadenoma does occur and can present considerable diagnostic problems on frozen section biopsy examinations (Azzopardi, 1979). However, in these conditions, the infarction is localized in contrast to the generalized involvement in anticoagulantinduced haemorrhagic infarction of the breast.

Flood et al. (1943) described a case of anticoagulant-associated gangrene of the breast which they termed as thrombophlebitis migrans disseminata. Haemorrhagic necrosis or infarction of the breast occurs usually 2-8 days after initiation of anticoagulant therapy with coumarin or indanedione derivatives. The pathological process appears to be irreversible and is not modified either by withdrawal of the drug or continuation of treatment with the same drug. In the pathogenesis of the lesion, aseptic ischaemic necrosis has been suggested although the extensive collateral blood circulation of the breast 


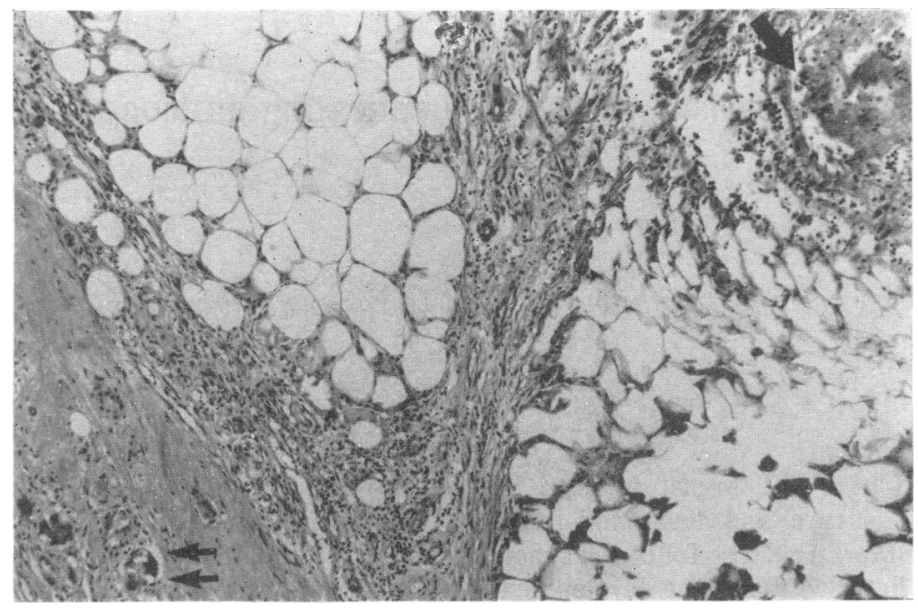

FIG. 1. Photomicrograph shows an area of total cell necrosis (single arrow) and lipogranulomatous reaction (double arrows) $(\mathrm{HE}, \times 200)$

does not support this view (Tong, 1971). Venous thrombosis and acute arteritic changes involving the small and medium sized arteries have been found in 3 cases described by Nudelman and Kempson (1966). In the present case, the authors could not demonstrate any such vascular changes. The possibility of hypersensitivity or allergic vasculitis seems to be rather remote because of the lack of further effect of the anticoagulant drug on continuation of treatment.

Skin lesions such as petechiae, ecchymoses and necrosis have been recorded as being induced by congeners of the coumarin group of anticoagulants. In the pathogenesis of these lesions, toxic effects of the drug on the dermovascular loop at the junction between the capillary and precapillary arterioles have been suggested (Nalbandian et al., 1965). The present authors feel that the lesion is multifactorial in origin. Direct effect of the oral anticoagulant on the cutaneous vasculature, in conjunction with alteration of platelet aggregation (Poller, Thomson and Priest, 1969) and trivial trauma to the breast initiate the disease process. Rupture of capillary blood vessels ensues and results in interstitial $\vec{c}$ haemorrhages. The latter would then lead to com- $\frac{\rho}{c}$ pression of venules and arterioles resulting in haemorrhagic infarction of the breast.

\section{References}

Azzopardi, J.G. (1979) Problems in Breast Pathology, edn, p. 44. W. B. Saunders, Philadelphia.

Flood, E.P., Redish, M.H., Bociek, S.J. \& Shapiro, $\overrightarrow{\mathbf{S}} . \underline{\Xi}$ (1943) Thrombophlebitis migrans disseminata: report of a case in which gangrene of a breast occurred. New York State Journal of Medicine, 43, 1121.

HAAGENSEN, C.D. (1971) Diseases of the Breast, 2nd edn, pp. 213, 215. W. B. Saunders, Philadelphia.

Nalbandian, R.M., Mader, I.J., Barrett, J.L., Pearce, O J.F. \& RUPP, E.C. (1965) Petechiae, ecchymoses and necrosis of skin induced by coumarin congeners. Journal of the American Medical Association, 192, 603.

Nudelman, H.L. \& Kempson, R.L. (1966) Necrosis of the breast-a rare complication of anticoagulant therapy. American Journal of Surgery, 111, 728.

Poller, L., Thomson, J.M. \& Priest, C.M. (1969) Coumarin therapy and platelet aggregation. British Medical Journal. $1,474$.

ToNG, D. (1971) Haemorrhagic necrosis of the breast complicating anticoagulant therapy and mitral stenosis. British Journal of Surgery, 58, 624. 